\title{
Coupling Schemes in Terahertz Planar Metamaterials
}

\author{
Dibakar Roy Chowdhury, ${ }^{1}$ Ranjan Singh, ${ }^{1}$ Antoinette J. Taylor, ${ }^{1}$ Hou-Tong Chen, ${ }^{1}$ \\ Weili Zhang, ${ }^{2}$ and Abul K. Azad ${ }^{1}$ \\ ${ }^{1}$ Center for Integrated Nanotechnologies, Materials Physics and Applications Division, Los Alamos National Laboratory, \\ Los Alamos, NM 87545, USA \\ ${ }^{2}$ School of Electrical and Computer Engineering, Oklahoma State University, Stillwater, OK 74078, USA
}

Correspondence should be addressed to Dibakar Roy Chowdhury, dibakar@lanl.gov and Ranjan Singh, ranjan@lanl.gov

Received 2 October 2011; Accepted 2 December 2011

Academic Editor: Zhaolin Lu

Copyright (c) 2012 Dibakar Roy Chowdhury et al. This is an open access article distributed under the Creative Commons Attribution License, which permits unrestricted use, distribution, and reproduction in any medium, provided the original work is properly cited.

\begin{abstract}
We present a review of the different coupling schemes in a planar array of terahertz metamaterials. The gap-to-gap near-field capacitive coupling between split-ring resonators in a unit cell leads to either blue shift or red shift of the fundamental inductivecapacitive $(L C)$ resonance, depending on the position of the split gap. The inductive coupling is enhanced by decreasing the inter resonator distance resulting in strong blue shifts of the $L C$ resonance. We observe the $L C$ resonance tuning only when the split-ring resonators are in close proximity of each other; otherwise, they appear to be uncoupled. Conversely, the higher-order resonances are sensitive to the smallest change in the inter particle distance or split-ring resonator orientation and undergo tremendous resonance line reshaping giving rise to a sharp subradiant resonance mode which produces hot spots useful for sensing applications. Most of the coupling schemes in a metamaterial are based on a near-field effect, though there also exists a mechanism to couple the resonators through the excitation of lowest-order lattice mode which facilitates the long-range radiative or diffractive coupling in the split-ring resonator plane leading to resonance line narrowing of the fundamental as well as the higher order resonance modes.
\end{abstract}

\section{Introduction}

The interaction of electromagnetic waves with unstructured bulk matter is mainly governed by the inherent chemistry of material, and its properties are usually described in terms of permittivity and permeability. Recently developed electromagnetic metamaterials allow engineered material at the unit cell level, which has enabled novel methods to manipulate the electromagnetic properties [1]. Metamaterials have attracted a tremendous amount of interest because of their exotic properties such as negative refractive index [2], perfect focusing [3], cloaking [4, 5], and resonance modulation in the active and passive modes [6-29]. The electromagnetic responses offered by metamaterials are unique and are not found in naturally occurring materials. The fundamental building blocks of metamaterials are mainly comprised of a metallic split-ring resonators (SRRs) fabricated on a die-lectric/semiconducting substrate. The operation of most metamaterial devices depends on the designs employed to control the fundamental resonances of SRRs, since the major tuning of material permittivity and permeability is enabled by these resonances [6-29]. Apart from the single-SRR approach, several research groups have explored lateral coupling between nearest neighbor SRRs and its effects on the resonances [30-42]. It turns out that one of the prominent schemes to passively tune the metamaterial resonances is achieved by changing either the intercell distance or the relative orientation of SRRs with respect to each other. These studies revealed that the laterally coupled mechanisms provide an extra degree of freedom for the manipulation of metamaterial responses. Moreover, the terahertz frequency range offers a convenient platform for these studies, since sample fabrication is straightforward, and terahertz time-domain spectroscopy (THz-TDS) provides a phase-coherent characterization technique.

\section{Coupling Mechanisms in Planar Metamaterials}

The short-range coupling in metamaterials occurs via the electric and magnetic fields due to the close proximity of 
the nearest neighbor resonators. The electric field couples through the gaps of the SRRs, while the magnetic field couples through the circumference, producing a red shift or blue shift of the resonance, depending on the nature of near-field coupling. We employed two different SRR design schemes to experimentally probe the effect of such coupling. In the first scheme, the density of the resonators is kept fixed, while the nearest neighbor interaction between two SRRs in the unit cell is probed as a function of their separation and mutual orientation. In the second type of experimental design, the effect of packing density is also taken into account. In this case, the number of SRRs excited by the incident beam varies from one sample to another as the resonators are collectively brought closer to each other. Thus, these results highlight the coupling behavior in metamaterials with low-, medium-, and high-density SRR arrays.

Recently, it has been demonstrated that near-field electric and magnetic coupling is not the only mechanism by which the SRRs in a planar arrangement remains coupled to each other [38-40]. In addition, there is also long-range radiative coupling as demonstrated with inductive-capacitive (LC) resonances by Singh et al. [39] and for higher-order resonance by Bitzer et al. [38]. This long-range diffractive coupling occurs when the lattice periodicity of the SRR array matches the wavelength of incident light at the fundamental $L C$ or higher-order resonances, leading to a tremendous reduction in radiation damping that enhances the quality factor of the coupled resonances.

\subsection{Capacitive Coupling between SRR Pairs in a Planar Array.} In this section, we discuss the effect of different gap-togap coupling schemes on the fundamental $L C$ resonances. The metamaterial samples studied were fabricated on semiinsulating GaAs substrates by photolithography methods, electron beam deposition $200 \mathrm{~nm}$ thick Au using $10 \mathrm{~nm}$ thick $\mathrm{Ti}$ as adhesion layer, and followed by a lift-off process. In the fabricated samples, each of the metamaterial unit cells contains two SRRs oriented with their gaps facing toward each other, as shown in Figures 1(a)-1(d) [29]. The samples were characterized in transmission using THz-TDS in a confocal geometry [43], using a bare GaAs substrate as the reference. The incident $\mathrm{THz}$ beam was linearly polarized with the electric field oriented parallel to the gap-bearing side, as shown in Figure 1(d).

In Figures 1(a) and 1(b), the two SRRs are individually identical, and their mutual coupling is controlled by varying their separation. As the separation between the resonators decreases from MM1 to MM2, a blue shift of metamaterial resonance is observed (Figure 2(a)). The coupling can also be controlled by asymmetry through displacing one of the gaps but keeping the other gap at the center of the gap-bearing arm (MM3 in Figure 1(c)), or by displacing both the gaps but in the opposite directions (MM4 in Figure 1(d)).

While displacing the gaps in MM3 and MM4 from their original position in MM2, the separation between SRRs within the unit cell is kept fixed at $2 \mu \mathrm{m}$. We observe a red shift accompanied by a reduction in the strength of the
$L C$ resonance for MM3 as compared to MM2 due to the asymmetry and a further red shift and strength reduction in MM4 (Figure 2(b)).

The fundamental $L C$ resonance mode arises because of the electric currents oscillating around the full circumference of the SRR loop. The electric currents are excited along the SRR arms by the incident electric field due to the asymmetric arm length of the individual elements of SRR unit cells. The position of the anticipated $L C$ resonance frequency can usually be described by a simple expression, $\omega_{L C}=1 / \sqrt{L C}$, where $L$ and $C$ are the effective inductance and capacitance of the individual SRR. In order to have better understanding of the physical mechanisms related to the resonant frequency shift from MM1 to MM4, we simulated the electric and magnetic field distributions using the numerical solver CST Microwave Studio [44]. Simulation results reveal that the magnetic field distributions for the coupled resonators remain almost similar to the uncoupled SRRs, and therefore, do not contribute to the resonance frequency shift significantly. However, the electric fields within the SRR pairs, as shown in Figure 3, clearly go through significant changes for the various coupled resonators. When the SRRs are far apart and SRR gaps are aligned, as in the case of MM1, fringing fields around the gap-bearing side contribute significantly to the overall capacitance of the individual resonators, as seen from the electric field distribution in Figure 3(a). As the separation between SRRs is reduced, these fringing fields increasingly couple the individual responses of the rings. Coulomb repulsion occurs through the fringing fields between like charges in the paired rings, thereby opposing charge accumulation near the gap resulting in a reduction of the fringing capacitance in both rings, as shown in Figure 3(b). This is responsible for the observed blue shift of the resonance frequency in MM2 as compared to MM1. When one gap is displaced completely towards the corner, as in the case of MM3, the Coulomb interaction between charges in one of the arms of the paired rings becomes attractive, thereby increasing the net electric flux density, as shown in Figure 3(c). This produces additional capacitance between the rings causing a red shift in the resonance.

For the sample MM4, both the gaps are displaced towards the corner, therefore, enhancing the Coulomb attraction between the opposite charges between the paired rings by maximum extent. This effect gives rise to a larger value of the coupling capacitance, resulting in maximum red shift of the $L C$ resonance. The red shift in MM3 and MM4 is accompanied by a weakening of the resonance, as revealed by the increased resonant transmission minimum. This is because of the weak excitation of SRRs with displaced gaps by the external electric field. Therefore, two apparently independent and simultaneous effects are observed: strong capacitive coupling between SRRs causing resonance frequency shifts and symmetry breaking in the individual rings causing variation of the resonance strength. The larger the capacitive coupling between the paired rings, the larger the resonance frequency shift. Similarly, the larger the asymmetry of the rings, the weaker the $L C$ resonance strength due to the weak coupling of the incident $\mathrm{THz}$ radiation with the SRRs. 


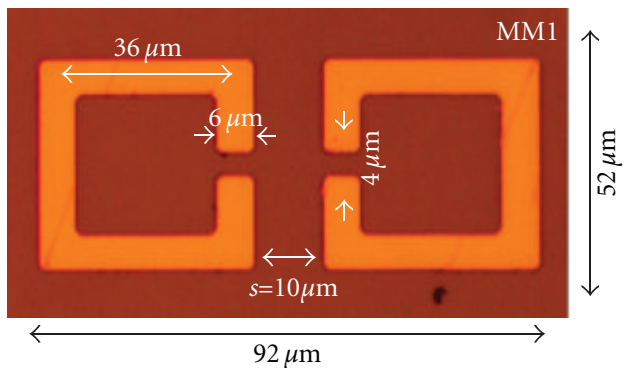

(a)

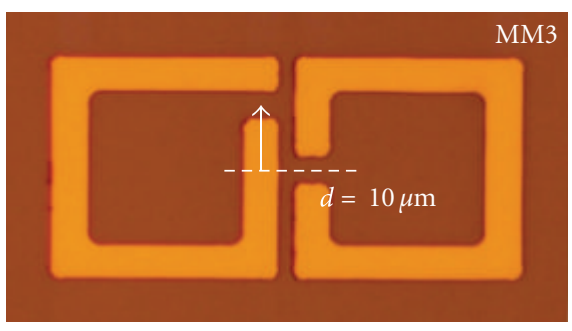

(c) (b)

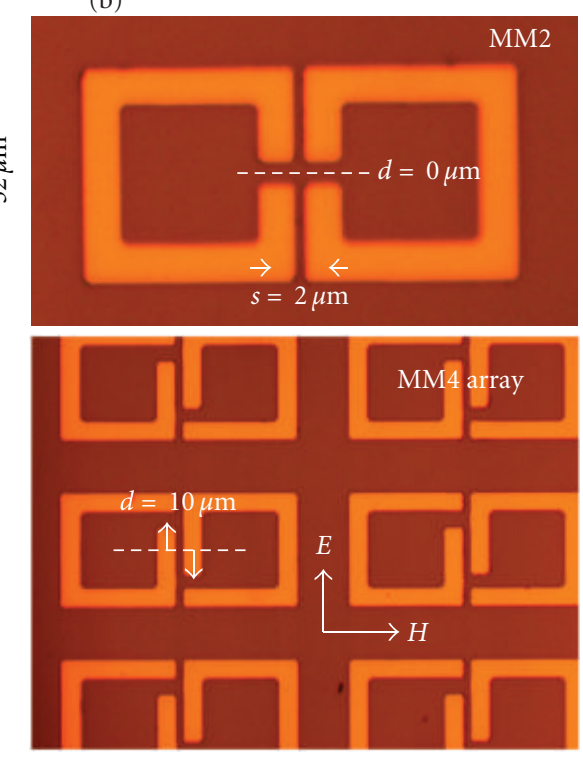

(d)

FIGURE 1: Optical microscope images of the fabricated metamaterial samples. (a) In the unit cell with symmetric gap-to-gap coupled SRRs, the separation is either (a) $10 \mu \mathrm{m}$ (MM1) or (b) $2 \mu \mathrm{m}$ (MM2). (c) The asymmetric SRRs with one gap displaced from the center (MM3) and (d) the SRR array where both gaps are displaced in the opposite direction from the center (MM4). The dimensions of the unit cells and their features are indicated in the figure. The unit cell periodicity for all the metamaterial samples is identical.

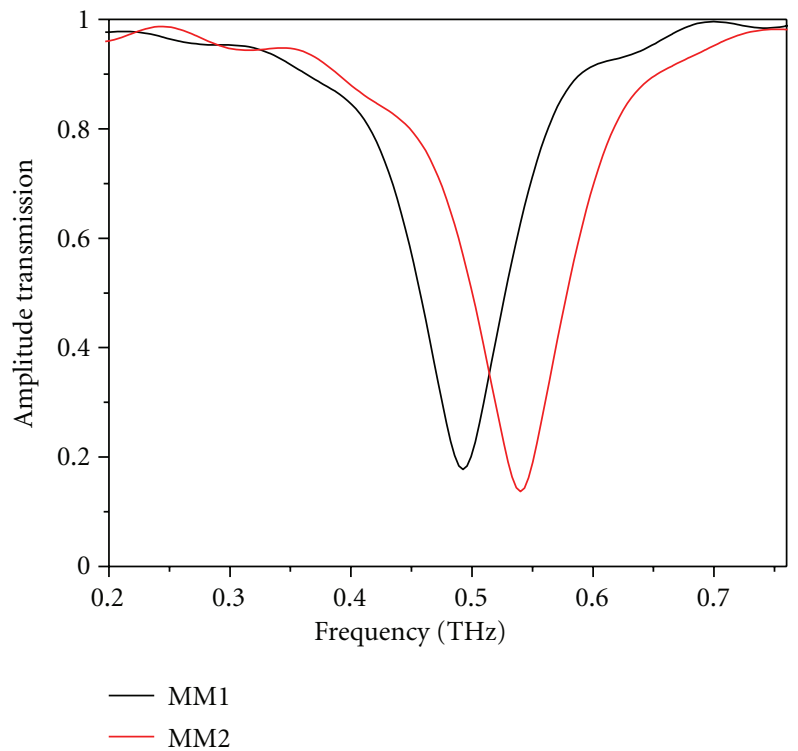

(a)

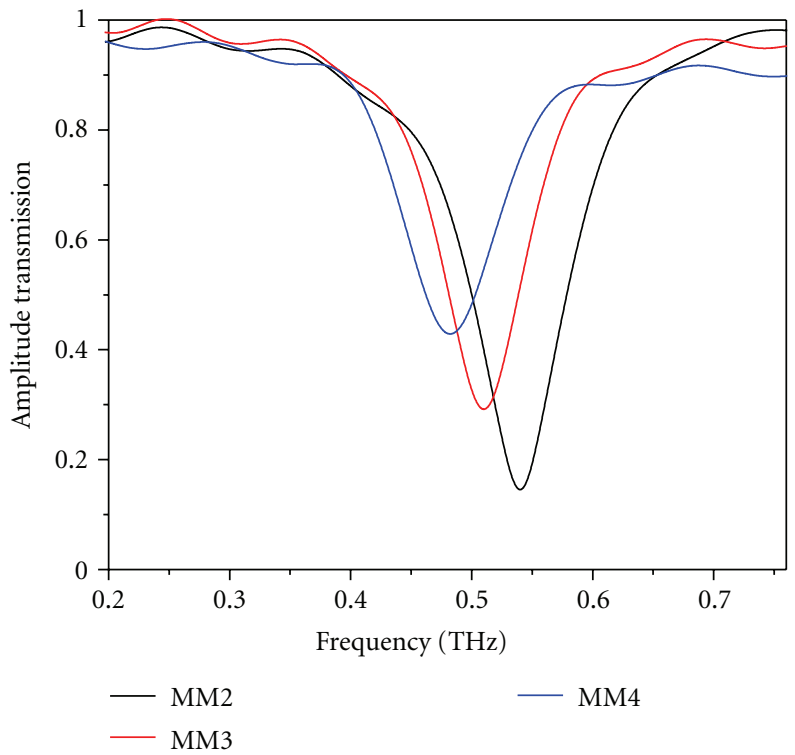

(b)

FIGURE 2: Measured THz electric field amplitude spectra when transmitting through the metamaterial samples for (a) MM1 and MM2 (b) and MM2, MM3, and MM4.

2.2. Inductive Coupling in the Metamaterial Array. The effect of strong inductive coupling was probed in terahertz SRRs by maintaining a fixed orientation and the capacitive gaps facing upwards in all samples, which minimizes the effect of the capacitive coupling [39]. Thus, the metamaterials are designed to have different periodicity from one sample to another. Nine sets of samples were prepared with periodicity of $P=200,150,100,60,50,40,35,30$, and $25 \mu \mathrm{m}$, keeping the geometrical dimensions of the SRR fixed. Figure 4(a) shows the metamaterial sample with $P=25 \mu \mathrm{m}$. The dimensions of SRR were chosen such that its $L C$ resonance wavelength is $\lambda \sim 200 \mu \mathrm{m}$ (i.e., $1.5 \mathrm{THz}$ ) on the silicon substrate. Different periodicities in samples were designed so that the resonance wavelength to lattice constant ratio $\lambda / P$ varies from a value 

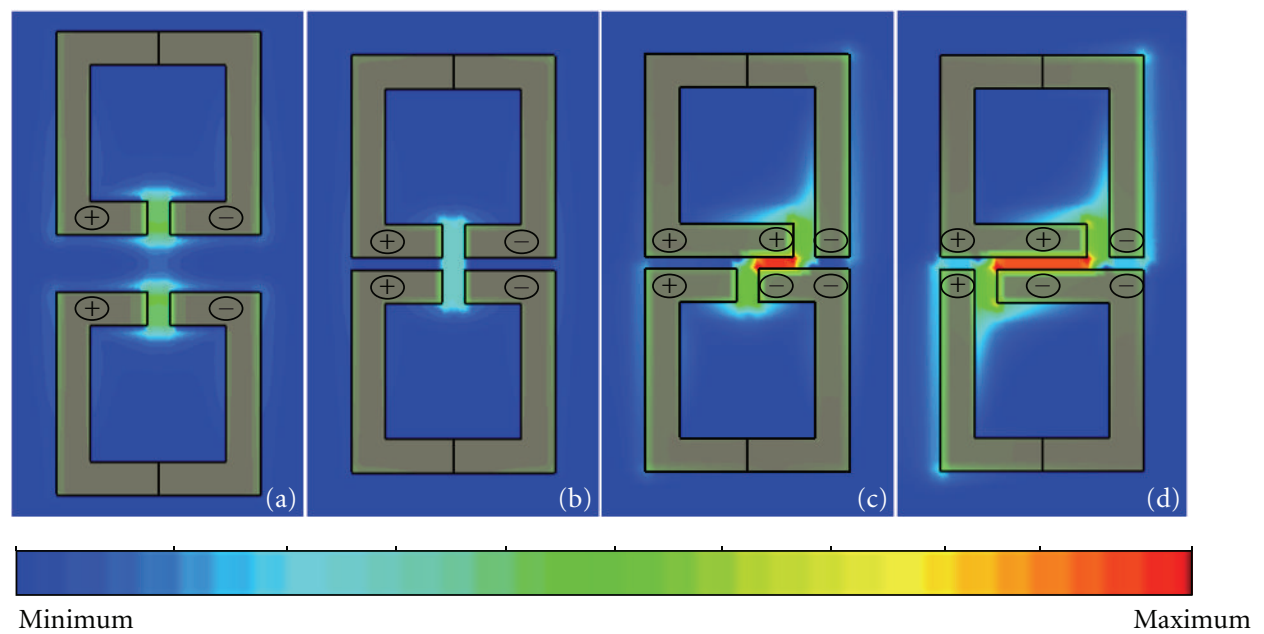

FIGURE 3: The numerically simulated electric field strength distributions for the coupled SRRs: (a) MM1 (b), MM2 (c), MM3 (d), and MM4. The electric field strength is indicated by the color map at the bottom. The polarity of the induced electronic charges on the SRR arms is indicated in the figure.

of $\lambda / P=1$ for $P=200 \mu \mathrm{m}$ to $\lambda / P=8$ for $P=25 \mu \mathrm{m}$. Each metamaterial sample is excited by an incident terahertz beam with the focal diameter of $3.5 \mathrm{~mm}$, with which the number of excited SRRs is gradually increased from 960 for $P=$ $200 \mu \mathrm{m}$ to 61,500 for $P=25 \mu \mathrm{m}$. This enables the study of the inter-SRR packing-dependent coupling and its impact on the overall collective resonant response in $\mathrm{THz}$ transmission.

The measured resonant transmission spectra are plotted in Figure 4(b). The evolution of the $L C$ resonance was observed at around $1.5 \mathrm{THz}$. The resonance was fairly weak for the 200,150 , and $100 \mu \mathrm{m}$ periodicities. A strong resonance feature is observed for the metamaterial sample with period of $60 \mu \mathrm{m}$, and then a gradual broadening of the resonance was seen for smaller periodicities or higher packing densities.

The dominant coupling mechanism among SRRs at the $L C$ resonance with increasing packing density is mainly magnetic/inductive in nature. The increase in the packing density leads to spatially closer magnetic loops strongly coupled to each other. When this happens, the mutual inductance between SRRs tends to reduce the self-inductance of the SRR loop leading to a reduced effective inductance and thus an increase or blue shifting of the $L C$ resonance frequency. Thus, the stronger is the inductive coupling between the SRRs, the higher the resonance frequency will be, since each SRR due to mutual coupling reduces the magnetic flux of its neighbors. The experimental data shows that stronger (high density) or weaker (low density) magnetic coupling leads to lowering of $Q$ factor of SRRs as shown in Figure 4(c). Further, there seems to be an optimal packing density with periodicity of $60 \mu \mathrm{m}$ where the SRRs are coupled to yield the sharpest $L C$ resonance due to diffractive coupling. This coupling mechanism is explained in detail in the next section.

2.3. Long-Range Diffractive Coupling in Metamaterial Array. In this section, we describe the long-range radiative coupling encountered in the experiment described in the previous section. We highlight that the inter-SRR distance and its number density or periodicity can control the $Q$ factor and the strength of the $L C$ resonance of SRRs. The measured amplitude transmission in Figure 4(b) shows that the $L C$ resonance at $60 \mu \mathrm{m}$ periodicity yields the narrowest resonance linewidth. Coincidentally, it also corresponds to a $\lambda / P$ ratio of 3.33 which is the refractive index of the silicon substrate on which the SRRs are fabricated. Thus, we observe a strong suppression of radiative losses and thus a sharp enhancement of the $Q$ factor at a certain critical periodicity, $P_{c}$, that exactly matches the SRR resonant wavelength in the silicon substrate, that is, $P_{c}=\lambda_{L C} / n$, where $\lambda_{L C}$ is the $L C$ resonance wavelength in vacuum, and $n$ is the refractive index of the substrate material. The sharpening of $L C$ resonance is mainly due to Wood's anomaly or the diffractive lattice mode corresponding to the critical periodicity, the resonant wavelength, and the substrate index [38-40,45]. When the periodicity is further reduced, a gradual decline in $Q$ factor of the fundamental SRR resonance is observed in Figure 4(c).

The narrowest resonance occurs when the incident light field corresponds to diffractive grating order $(i, j)$ change from evanescent to radiative, $P_{c}=\sqrt{\left(i^{2}+j^{2}\right)} \lambda / n$; here $n$ is the refractive index of the substrate in use, $(i, j)$ are the diffraction grating orders, and $\lambda$ is the resonance wavelength. In the experiment, $P_{c}=60 \mathrm{um}$ corresponds to the lowestorder $(1,0)$ diffractive lattice mode. Every time the lowestorder lattice mode matches either the lower-order fundamental $L C$ resonance or higher-order resonances, a sudden sharpening of the resonance will occur. The narrowing of the resonance in an optimally packed metamaterial can also be best understood by the suppression of radiation damping due to the confinement or trapping of electromagnetic fields in the metamaterial plane. This mechanism is based on diffractive coupling during which the diffracted fields are trapped in the metamaterial lattice, and each SRR radiatively couples to the neighboring SRR. The lower $Q$ factor for larger 


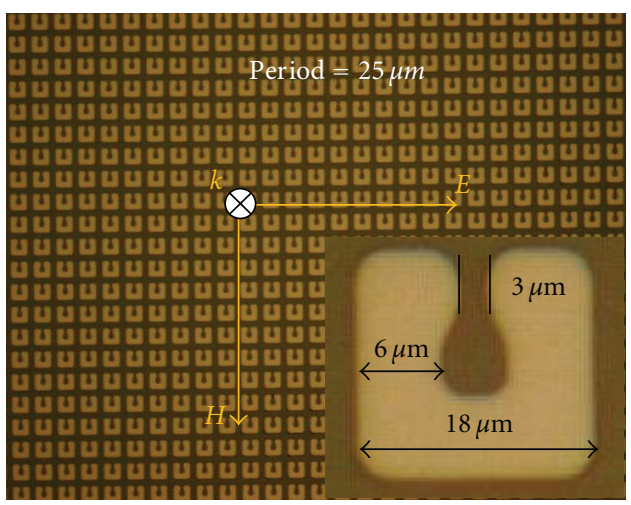

(a)

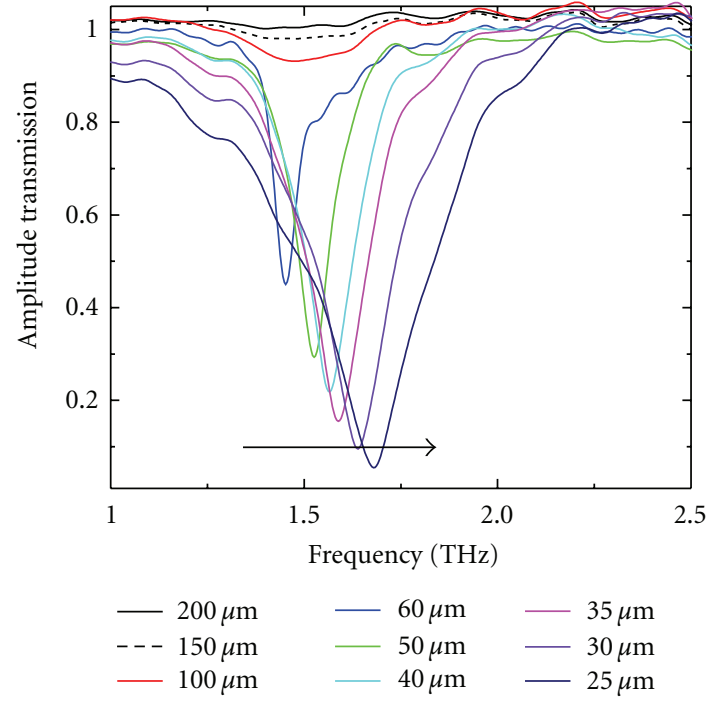

(b)

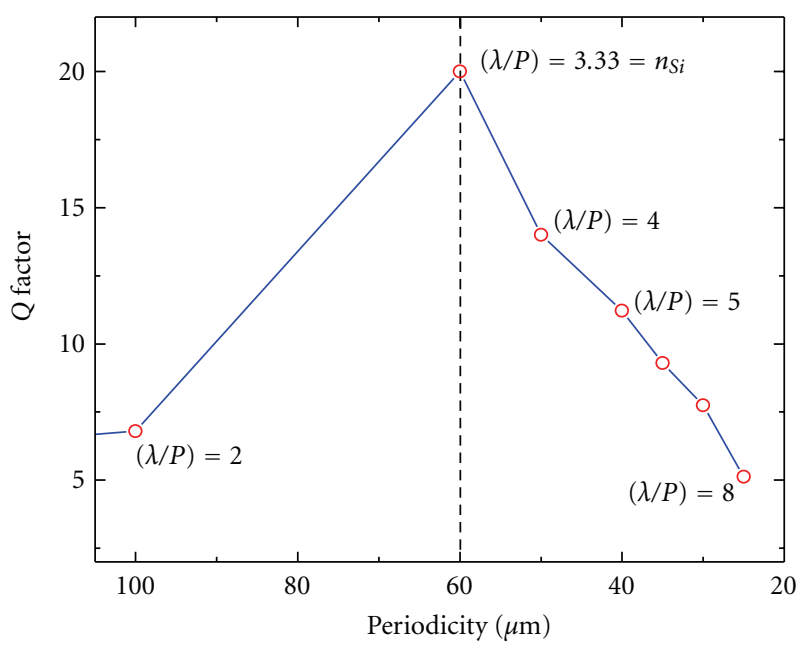

(c)

FIGURE 4: (a) Microscopic image of the highest-density metamaterial sample. The SRRs are comprised of $200 \mathrm{~nm}$ thick Al on $640 \mu \mathrm{m}$ thick n-type silicon substrates. (b) Measured transmission spectra of metamaterials with different periodicities. (c) Quality factor extracted from the measured transmission for different period metamaterials.

periods is associated to an enhanced radiation damping. The lower $Q$ factor for smaller periods is mainly due to the mismatch with the lattice mode during which the radiated fields of the coherently coupled SRRs interfere destructively in the plane of the metamaterial, leading to higher radiative losses and increase of nonradiative losses due to a capacitive and inductive coupling.

Tailoring the periodicity of metamaterials will allow the design of frequency-selective surfaces with an adjustable $Q$ factor for metamaterials across the entire electromagnetic spectrum optimized for various applications. This finding is important for the development of metamolecule density and substrate-refractive-index-tuned $\mathrm{THz}$ devices and components.
2.4. Capacitive and Inductive Coupling for Different Orientation and Separation in Nearest-Neighbor SRRs. In this section, we probe scenarios where neighboring split rings have their gap either on the same or on opposite sides with the change in periodicity [37]. The lowest-order odd eigenmode and the lowest-order even eigenmodes for two different excitations are marginally affected, whereas the higher-order eigenmode experiences a significant spectral reshaping $[37,46]$ due to a coherent interaction.

We designed metamaterials composed of three different supercells each containing four SRRs. The geometrical arrangement of the SRRs in each supercell is shown in Figure 5. In the first sample, all SRRs have their gap on the same side. In a second sample, the SRRs are arranged 

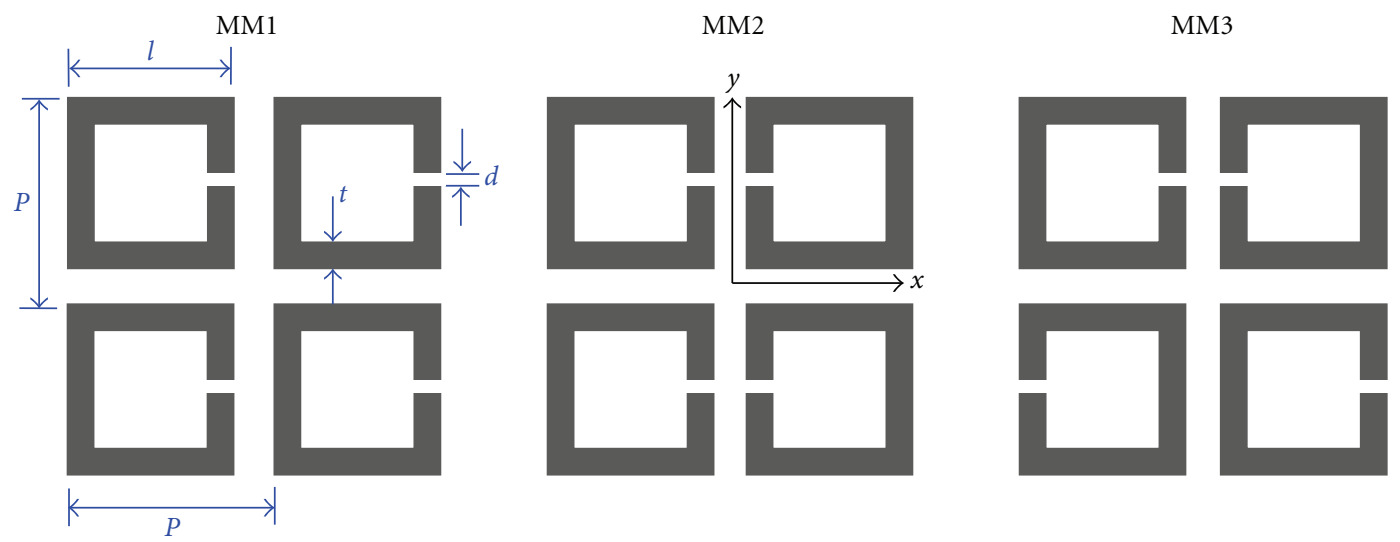

FIGURE 5: Unit cell of the three types of differently oriented split-ring resonators. The dimensions of all SRRs are $t=6 \mu \mathrm{m}, l=36 \mu \mathrm{m}$, and $d=2 \mu \mathrm{m}$. In the design, the period $P$ is varied.

with vertical mirror symmetry. In a third sample, the SRRs have the same orientation of their gaps along the diagonal directions of the supercell. In this work, the effects of the orientation on the spectral position and the width of each resonance are investigated. It was observed that control over the orientation of the SRRs and the periodicity enables extremely sharp features in the spectrum that are promising candidates for various practical applications such as sensing devices.

Simulations were done with the Fourier modal method [47] using the structure parameters shown in Figure 6 where only the periodicity parameter " $P$ " was swept from $P=50 \mu \mathrm{m}$ to $P=39 \mu \mathrm{m}$. Figure 6 shows the transmission for the three configurations as a function of period and frequency. In all cases, the incident $E$-field is polarized along the gap-bearing arms in the $y$-axis direction as shown in MM2. The main effects observed in the simulations are the impact of each configuration, the impact of the periodicity for each configuration on the spectral positions and the widths of the resonance, and the occurrence of coherent phenomena once SRRs are closely spaced. It can be clearly seen that the fundamental mode $L C$ resonance at $0.5 \mathrm{THz}$ does not undergo significant change in resonance position or resonance linewidth, but the higher-order dipole resonance undergoes a huge spectral change at about $1.5 \mathrm{THz}$.

Between MM1 and MM2, as the periodicity is reduced, the resonance at $1.5 \mathrm{THz}$ broadens in $\mathrm{MM} 2$, and the same resonance in MM3 splits and exhibits a very sharp spectral feature, shown by a white-dotted ellipse. Such large spectral reshaping prompted us to carry out the experiment in all of these configurations.

Six samples of planar SRRs (denoted in the following section as MM1-MM6) were fabricated. As shown in Figure 5 above, MM1-MM3 are single SRRs with lattice constant $P=50 \mu \mathrm{m}$. MM4-MM6 are single SRRs with the same symmetry as MM1-MM3 but with reduced periodicity of $P=39 \mu \mathrm{m}$. The measured amplitude transmission spectra of MM1-MM3 are shown in Figure 7(a). The two significant dips in transmission are traces from the two lowest-order

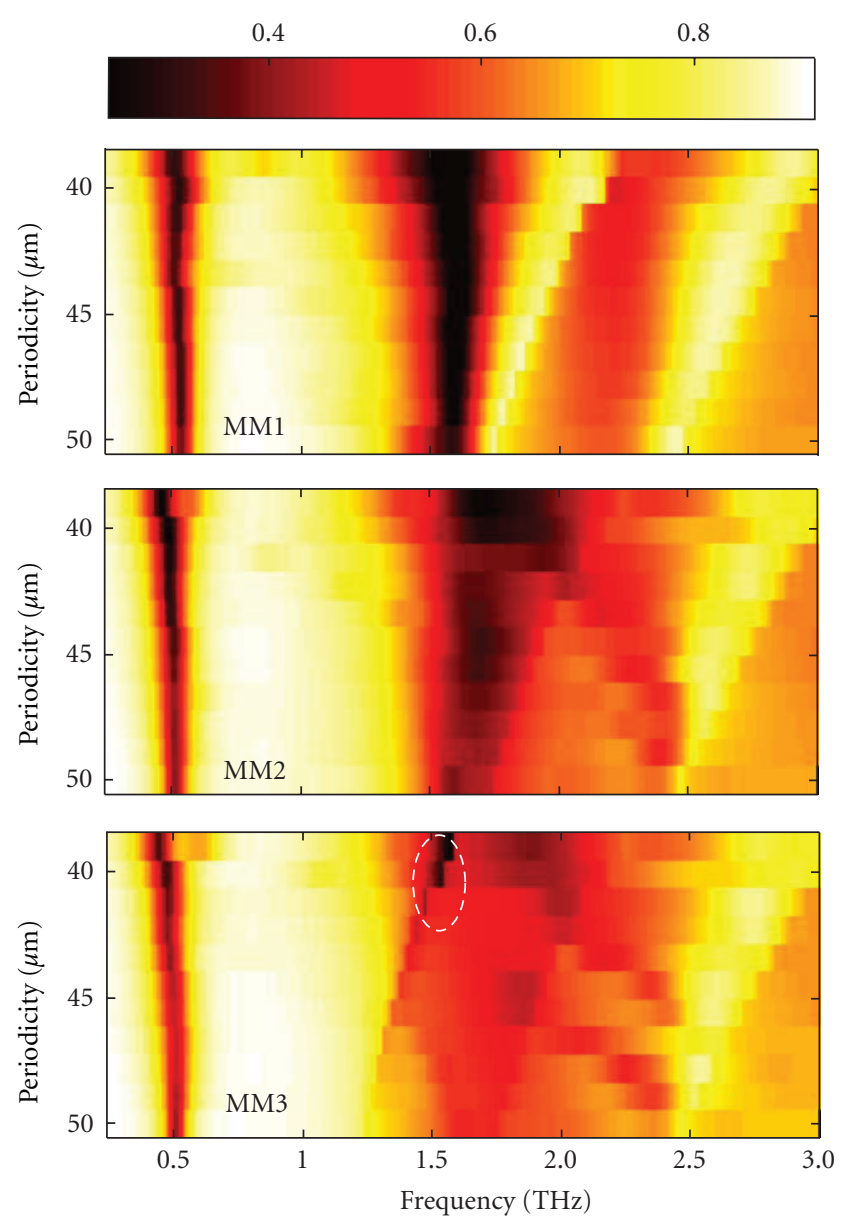

Figure 6: Simulated transmission spectra of the three differently oriented metamaterials with period, $P$, swept from $P=50 \mu \mathrm{m}$ to $P=$ $39 \mu \mathrm{m}$.

odd eigenmodes. Significant differences in the spectra are observed, depending on the orientation of the gap of neighboring SRRs. When comparing the resonances of MM2 with those of MM1, it can be seen that the $L C$ resonance at $0.5 \mathrm{THz}$ 


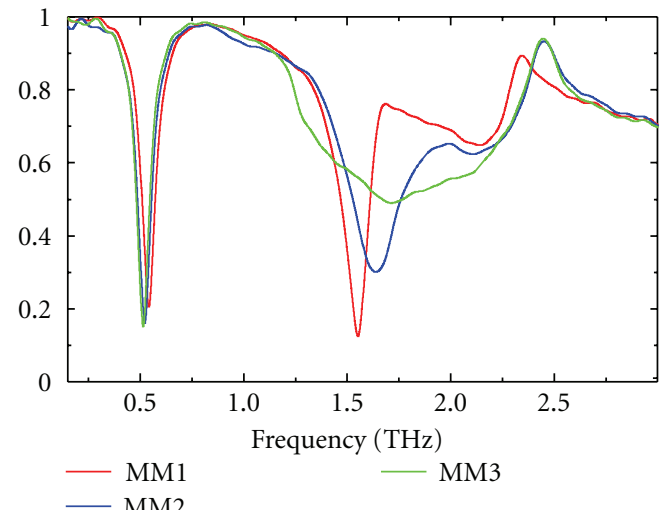

(a)

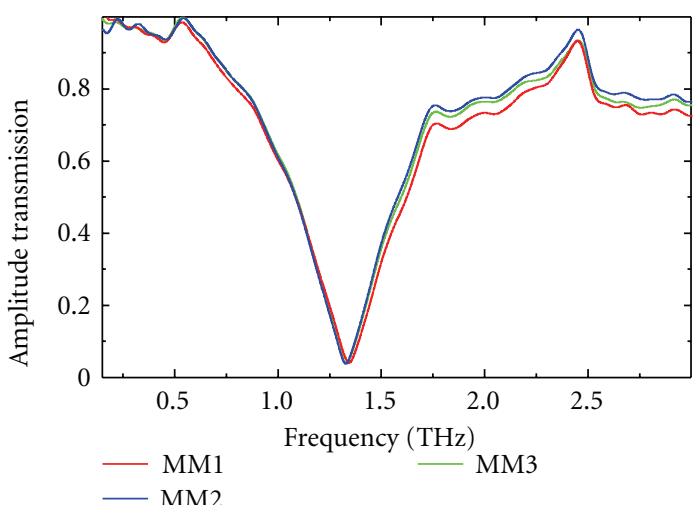

(c)

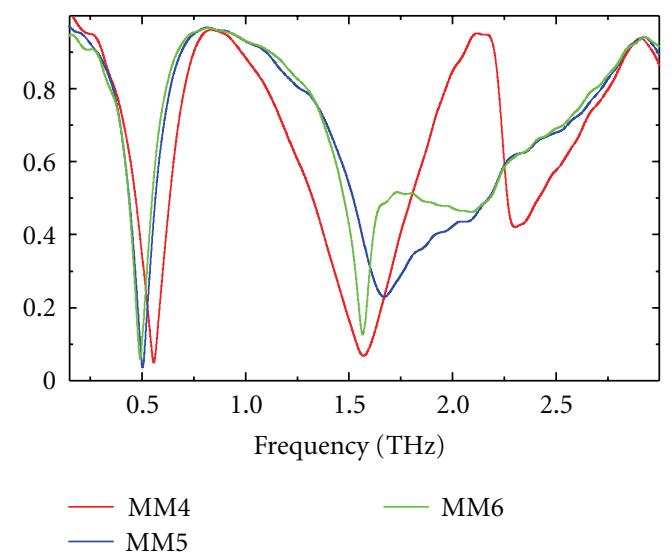

(e)

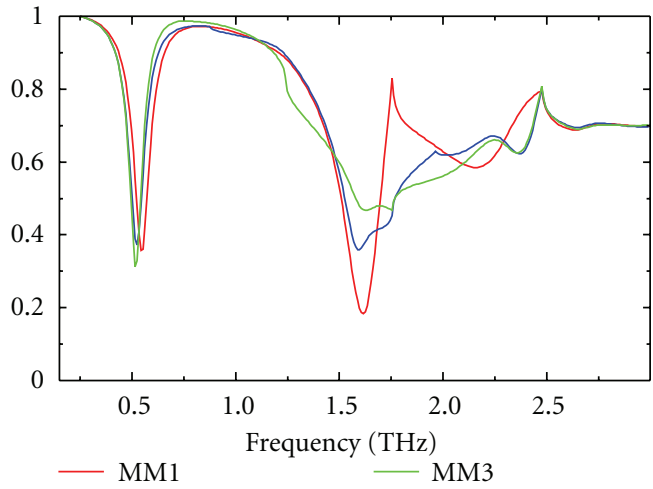

(b)

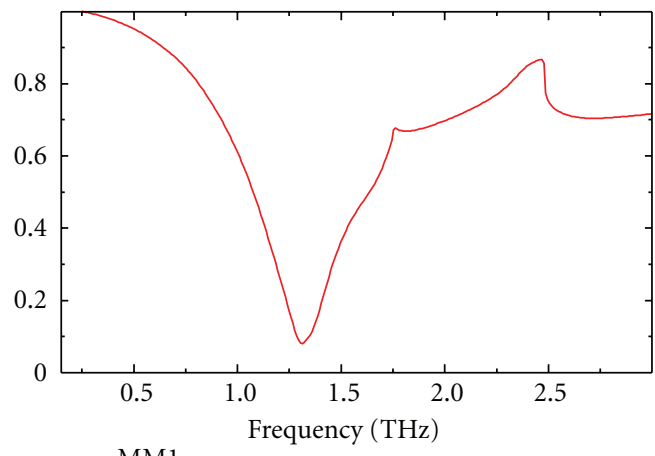

(d)

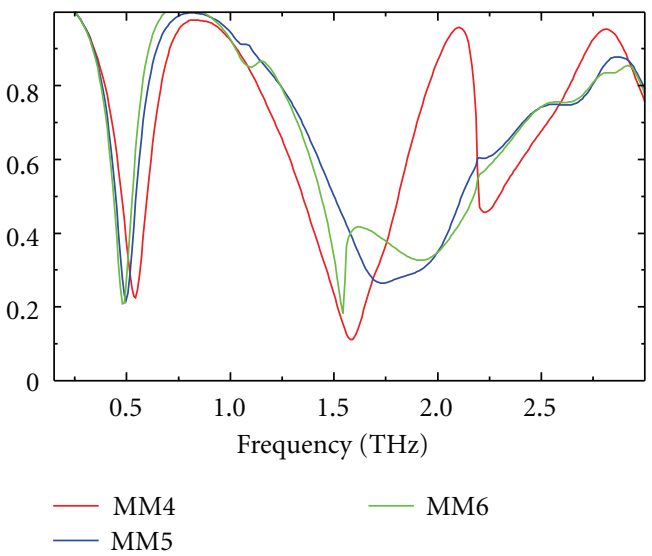

(f)

Figure 7: (a), (b) are measurement and simulations of differently oriented metamaterials, MM1, MM2, and MM3, for $P=50 \mu \mathrm{m}$ for excitation in $x$ direction, (c), (d) are the same but excitation in y direction, and (e), (f) are measurement and simulations for three differently oriented unit cells with period $P=39 \mu \mathrm{m}$.

is red shifted slightly. In contrast, the next higher-order odd resonance at $1.5 \mathrm{THz}$ broadens, blue-shifts by $84 \mathrm{GHz}$, and the transmission increases significantly in MM2. Furthermore, the orientation of the SRRs in the supercell for MM3 causes only a marginal additional red shift of the lowest-order eigenmode by $8 \mathrm{GHz}$ and nearly no change on transmission. The next higher-order resonances suffer from a severe broadening and a significant increase in transmission up to $49 \%$. The simulated spectra in Figure 7 (b) are in good agreement with the measurements except the amplitude transmission depth at the $L C$ resonance due to the limited resolution of our numerical code. For an incident $E$-field perpendicular to the gap-bearing arms, the response of MM1-MM3 is shown in Figure 7(c). Independent of the 


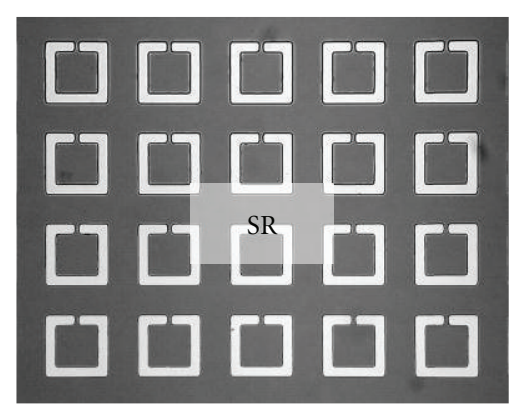

(a)

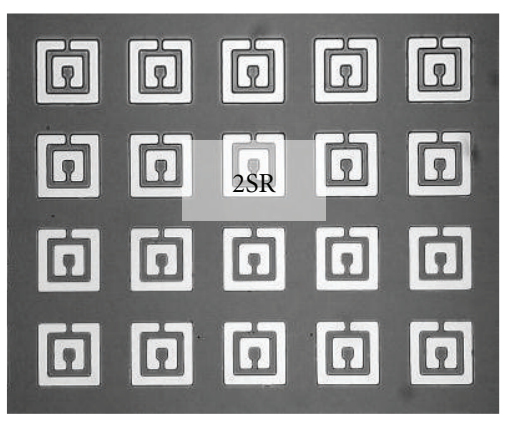

(c)

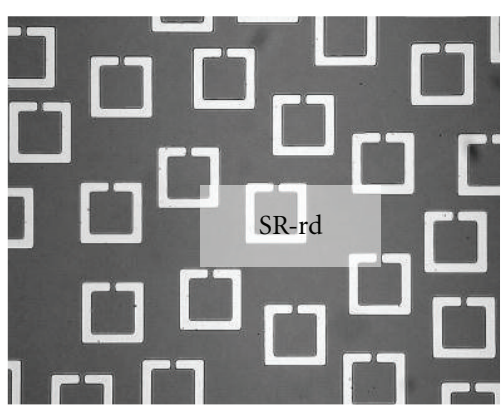

(b)

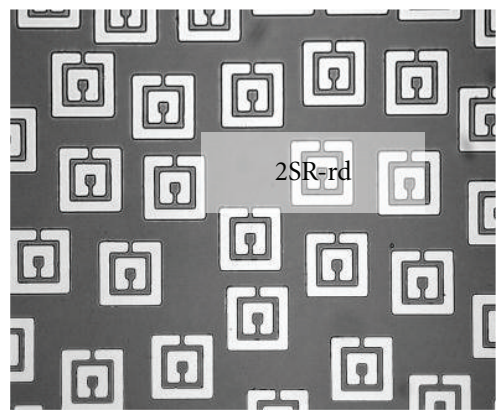

(d)

FIGURE 8: Microscopic images of the (a) periodic and (b) random single SRR array, (c) periodic and (d) random double SRR array. The dimensions of the outer ring are the same as in Figure 5 and of the inner smaller ring are the same as in Figure 4(a).

arrangement, all spectra show a strong resonance at $1.33 \mathrm{THz}$ and have nearly the same spectral dependence. Exactly the same observation is made in the simulation. This resonance is the lowest-order even eigenmode.

The experimentally measured transmission spectra for MM4-MM6 are shown in Figure 7(e). The incident $E$-field is polarized along $y$-axis. Due to reduced periodicity and high density of SRRs, we observe a different spectral behavior in comparison with the samples MM1-MM3. In MM4, there are three distinct resonances, the $L C$ resonance at $0.556 \mathrm{THz}$, the next higher-order odd eigenmode at $1.57 \mathrm{THz}$, and a third strong resonance at $2.3 \mathrm{THz}$. This third resonance, however, is a Wood's anomaly in the substrate. In MM5, the lowest-order odd eigenmode appears red-shifted and has a reduced linewidth when compared to MM4. The next higher-order odd eigenmodes are broadened and blueshifted. In MM6, the lowest-order odd eigenmode is again marginally further red-shifted. Finally, and potentially most interesting, the next higher-order odd eigenmode appears as an extremely sharp feature with a linewidth of only $69.5 \mathrm{GHz}$, resonating approximately at the same frequency as the higher-order odd eigenmode of MM4. The resonance has a high $Q$-factor of 18.5 . This high $Q$-factor is an essential property for the achievement of high sensitivity in planar THz MM sensors, as sensitivity increases with the sharpness of the spectral resonance feature. In Figure 7(f), the simulation agrees well with the measurements.

To explain the influence of the arrangement on the spectral properties of the lowest-order LC resonance mode, one may evoke analogies to transmission line theory. Placing SRRs like in MM2 and MM3 will cause an increase of the effective capacitance as the next neighbor in such situation provides a parasitic or mutual capacitance in parallel that would add up with the gap capacitance. The increase in effective capacitance causes the red shift of the $L C$ resonance.

For the lowest-order even eigenmode when the excitation field is along $x$ direction, placing the gap on either side of the SRR has no effect, as this mode is dominantly characterized by oscillating currents in the side arms of the SRR that have no gap. Therefore, modification of the resonance conditions for the SRR is neither observed nor expected.

For the third resonance which is the second-order odd eigenmode, the resonance shape and position depends on the chossen period and consequently on the absolute interSRR distance between the neighbors. By taking into account the fact that the resonance wavelength is comparable to the chosen interparticle separation, the coherent superposition of the scattered field by neighboring SRRs causes the strong modification of the transmission in this spectral domain. The formation of a subradiant and a superradiant type mode, depending on the interparticle distance and the gap orientation, causes a blue shift or a red shift of the resonance [43]. The superradiant (subradiant) mode also increases (decreases) the radiation losses of the excited eigenmode which leads to a broadening (narrowing) of the spectral resonance.

2.5. Coupling in the Randomly Distributed Metamaterial Array. In this section, we review the outcome of the introduction of positional disorder of SRRs in metamaterials on the quality factor of the fundamental $L C$ and higherorder dipole resonances. Several existing metamaterials are 


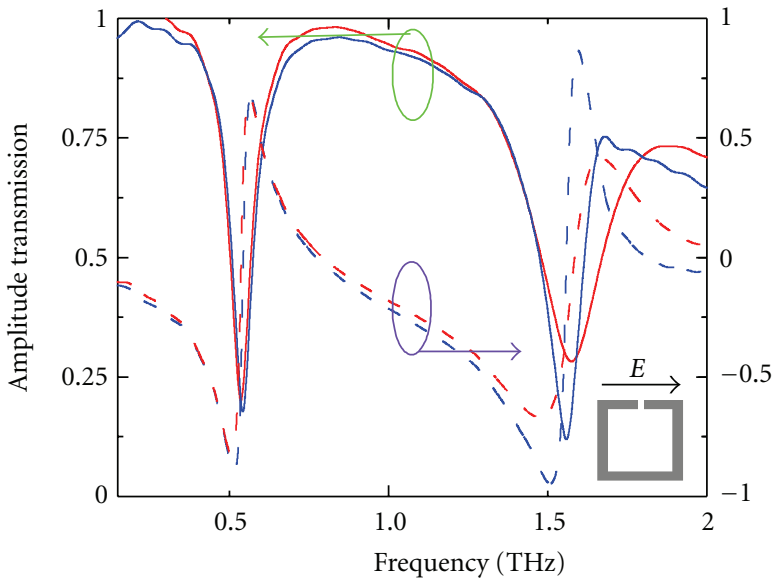

(a)

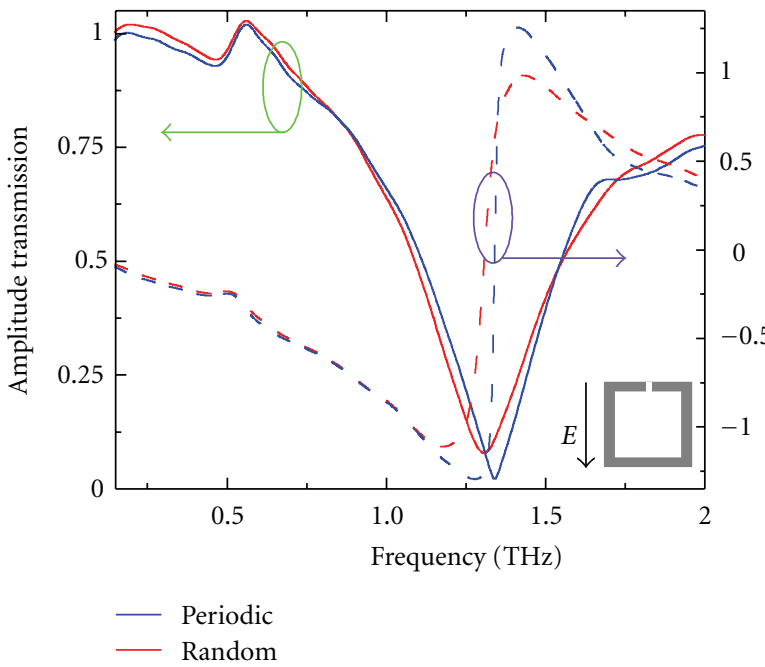

(b)

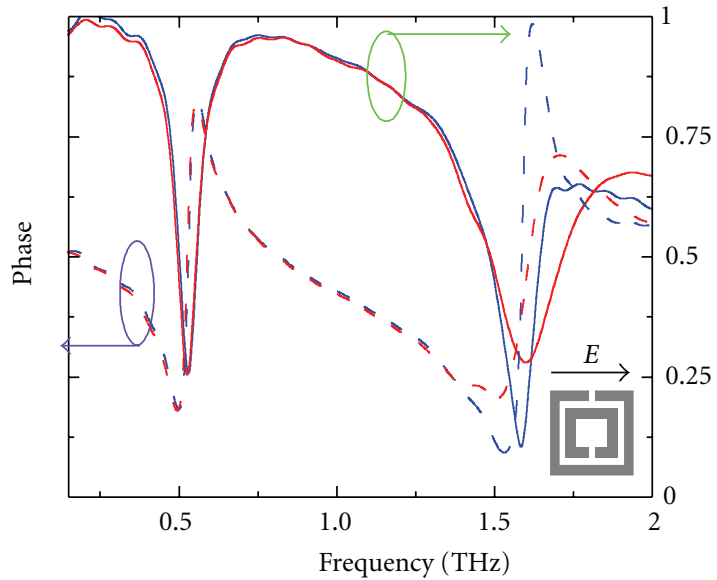

(c)

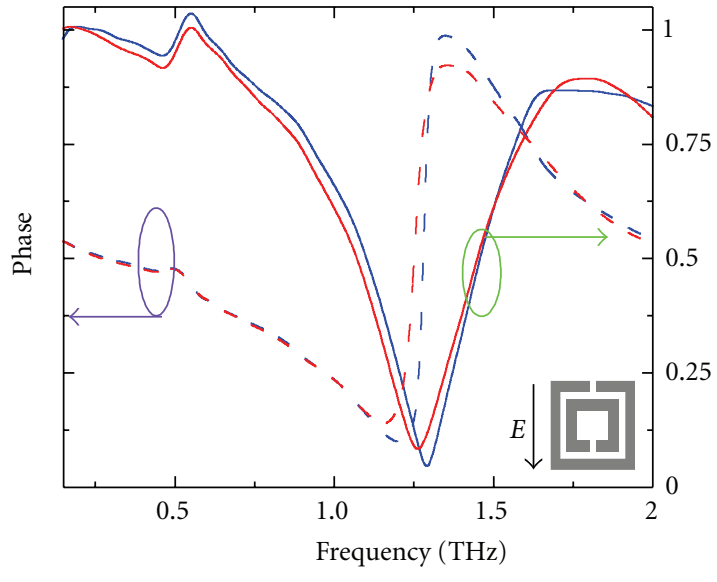

— Periodic

— Random

Figure 9: Measured (a) parallel and (b) perpendicular excitation of single-ring periodic and random resonators; (c), (d) are the same for double-ring resonators. The dash curves are the corresponding phase changes.

anisotropic in nature. However, for most practical applications across the entire electromagnetic spectrum, isotropic materials are required, and isotropic medium is sometimes based on randomly distributed cells in the volume of the host structure. Much of the previous work on metamaterials was focused on investigating the unique properties of wave propagation through periodic structures. There has been a tremendous desire to explore the collective behavior of SRRs when disorder is present in either their geometrical dimensions or periodic positioning on the host media [48-52].

The disorder has been studied in the single and the double split-ring resonators [50]. For single SRRs, only the outer ring with the same dimensions is retained. The disorder in arrangement was introduced by manually changing the lattice points of SRRs in a random fashion during the mask design. Each of the periodic SRRs was initially at a lattice position, $\vec{r}_{n}$, where $\vec{r}=x \vec{i}+y \vec{j}$, and individual SRRs were then displaced by $\pm \overrightarrow{\delta x}$ and $\pm \overrightarrow{\delta y}$ to introduce positioning disorder as shown in Figure 8. Thus, $\delta r=\delta x \vec{i}+\delta y \vec{j}$ is used to define the degree of randomness. In our case, $|\overrightarrow{\delta x}| \leq 32 \mu \mathrm{m}$ and $|\overrightarrow{\delta y}| \leq 32 \mu \mathrm{m}$, leading to a maximum disorder $|\overrightarrow{\delta r}| \leq$
$45.25 \mu \mathrm{m}$, which is equivalent to $85 \%$ of the periodicity, $P=$ $53 \mu \mathrm{m}$. The random SRRs retain the same number density as that of the counterpart periodic SRRs, giving the same metal volume filling fraction, $f=0.25$ for the single SRRs, $S R$ (periodic), and $S R$-rd (random), $f=0.33$ for the double SRRs, 2SR (periodic), and 2SR-rd (random).

For the random sample $S R$-rd in the parallel orientation, as shown in Figure 9(a), no change in the $L C$ resonance feature is observed compared to that of $S R$. The next higher resonance, however, blue-shifts by $16 \mathrm{GHz}$ with a linewidth broadening by $80 \mathrm{GHz}$ and a significant transmission modification from $12 \%$ to $28 \%$. In the perpendicular orientation, the lowest-order eigenmode of the random structure, $S R$ $r d$, red-shifts by $34 \mathrm{GHz}$ and broadens by $106 \mathrm{GHz}$, as shown in Figure 9(b). This behavior indicates that the dipole resonance is clearly affected by the positional disorder of SRRs.

Figures $9(\mathrm{c})$ and $9(\mathrm{~d})$ illustrate the frequency-dependent amplitude transmission of the double SRR samples, $2 S R$ and $2 S R-r d$. Similar effects are observed in the periodic and aperiodic double SRRs, as seen for the single SRRs. The $L C$ resonance of $2 S R$ and $2 S R-r d$ was found identical in all 
respects. The second-order odd eigenresonance at $1.58 \mathrm{THz}$ blue-shifted by $16 \mathrm{GHz}$ and broadened by $99 \mathrm{GHz}$, and the resonance minimum decreased by $17.5 \%$. The lowest even eigenmode red-shifts by $28.5 \mathrm{GHz}$ and broadens by $49 \mathrm{GHz}$. The corresponding phase change spectra are shown in all the figures.

The character of the $L C$ resonance is independent of randomness in the positioning of SRRs as its peak frequency and resonance strength remain unaffected. The response of the random SRRs at the $L C$ resonance is identical to that of their periodic counterpart as long as their number density remains constant by maintaining equal volume metal filling fraction. The inter-SRR interaction between the individual elemental SRR is negligible since the collective behavior of the periodic and the random metamaterials is just a direct addition of individual SRR contributions.

Unlike the $L C$ resonance, the dipole resonances in the random structures undergo a change compared to that of the periodic SRRs. This can be expected due to the different current profiles for the SRRs at $L C$ and dipole resonance frequencies. The second odd and lowest even eigenmode resonances in the transmission spectra of all the SRRs are due to linear currents in the SRR side arms and have similar current density distributions. Therefore, those resonances undergo similar spectral modification when disorder is introduced in the SRRs. The incident terahertz electric field excites plasmon oscillations of conduction electrons at the surface of individual metallic SRR arms that are parallel to the illuminating field, producing a collection of oscillating dipoles with a dipole moment. The dipole resonance is due to the dipole-dipole interaction between the SRR arms along the direction of the incident electric field. The plasmon energy and the resonance linewidth strongly depend on the interparticle distance. The dipole resonance wavelength in the metamaterial structures becomes comparable to the interparticle spacing, and as the distance between the dipoles is varied, there is formation of a coupled plasmon mode which alternates between superradiative and subradiative behavior modifying the resonance spectral width and causing frequency shifts. The dipole coupling among the randomized split ring resonators is highly complicated due to their random positioning with respect to each other and is influenced by a number of other factors, hence rendering a straightforward explanation impossible. In the periodic structures, the coupling between the dipoles is stronger compared to the randomly distributed SRRs since the interaction between the oscillating particles is partially cancelled by the disorder, which leads to weaker coupling among the dipoles and higher radiative damping. As a result, broadened and weaker plasmon resonances in the randomly scattered SRR metamaterials are observed. This is also due to inhomogeneous broadening that is caused due to the randomly scattered dipoles.

\section{Conclusion}

In conclusion, in-plane coupling of terahertz SRRs can manipulate and tailor the fundamental as well as higher-order resonances. The near-field coupling via the SRRs electric and magnetic field causes the fundamental mode resonance to red-shift and blue-shift, respectively. The mutual orientation and the separation plays dominant roles in tuning the higherorder dipolar resonance, leading to excitation of sharp subradiant resonances that can act like hotspots for sensing applications. The effect of packing density in SRRs can be very strong, enabling the design of tunable transmission and quality factor metamaterials. Long range radiative coupling also leads to resonance sharpening and electromagnetic energy trapping in the metamaterial lattice, and these longrange interactions are mediated by the fundamental-order diffractive mode of the periodic grating. Thus, the different coupling mechanisms in planar matamaterials could be exploited for the development of terahertz devices with various functionalities.

\section{Acknowledgments}

The authors thank C. Rockstuhl, J. F. O’ Hara, M. Walther, and X. Lu for their outstanding contribution and fruitful discussions in this work. Some of the work was partially supported by the U.S. National Science Foundation (NSF). This work was performed, in part, at the Center for Integrated Nanotechnologies, a U.S. Department of Energy, Office of Basic Energy Sciences user facility. Los Alamos National Laboratory, an affirmative action equal opportunity employer, is operated by Los Alamos National Security, LLC, for the National Nuclear Security Administration of the U.S. Department of Energy under Contract no. DE-AC5206 NA25396.

\section{References}

[1] J. B. Pendry, A. J. Holden, D. J. Robbins, and W. J. Stewart, "Magnetism from conductors and enhanced nonlinear phenomena," IEEE Transactions on Microwave Theory and Techniques, vol. 47, no. 11, pp. 2075-2084, 1999.

[2] R. A. Shelby, D. R. Smith, and S. Schultz, "Experimental verification of a negative index of refraction," Science, vol. 292, no. 5514, pp. 77-79, 2001.

[3] N. Fang, H. Lee, C. Sun, and X. Zhang, "Sub-diffractionlimited optical imaging with a silver superlens," Science, vol. 308, no. 5721, pp. 534-537, 2005.

[4] J. B. Pendry, D. Schurig, and D. R. Smith, "Controlling electromagnetic fields," Science, vol. 312, no. 5781, pp. 1780-1782, 2006.

[5] D. Schurig, J. J. Mock, B. J. Justice et al., "Metamaterial electromagnetic cloak at microwave frequencies," Science, vol. 314, no. 5801, pp. 977-980, 2006.

[6] W. J. Padilla, A. J. Taylor, C. Highstrete, M. Lee, and R. D. Averitt, "Dynamical electric and magnetic metamaterial response at terahertz frequencies," Physical Review Letters, vol. 96, no. 10, Article ID 107401, pp. 1-4, 2006.

[7] H. T. Chen, W. J. Padilla, J. M. O. Zide, A. C. Gossard, A. J. Taylor, and R. D. Averitt, "Active terahertz metamaterial devices," Nature, vol. 444, no. 7119, pp. 597-600, 2006.

[8] H. T. Chen, J. F. O’Hara, A. K. Azad et al., "Experimental demonstration of frequency-agile terahertz metamaterials," Nature Photonics, vol. 2, no. 5, pp. 295-298, 2008. 
[9] H. Tao, A. C. Strikwerda, K. Fan, W. J. Padilla, X. Zhang, and R. D. Averitt, "Reconfigurable terahertz metamaterials," Physical Review Letters, vol. 103, no. 14, Article ID 147401, 2009.

[10] Z. Tian, R. Singh, J. Han et al., "Terahertz superconducting plasmonic hole array," Optics Letters, vol. 35, no. 21, pp. 35863588, 2010.

[11] T. Driscoll, H. T. Kim, B. G. Chae et al., "Memory metamaterials," Science, vol. 325, no. 5947, pp. 1518-1521, 2009.

[12] R. Singh, E. Plum, W. Zhang, and N. I. Zheludev, "Highly tunable optical activity in planar achiral terahertz metamaterials," Optics Express, vol. 18, no. 13, pp. 13425-13430, 2010.

[13] J. Gu, R. Singh, Z. Tian et al., "Terahertz superconductor metamaterial," Applied Physics Letters, vol. 97, no. 7, Article ID 071102, 2010.

[14] B. Jin, C. Zhang, S. Engelbrecht et al., "Low loss and magnetic field-tunable superconducting terahertz metamaterial," Optics Express, vol. 18, no. 16, pp. 17504-17509, 2010.

[15] H. T. Chen, H. Yang, R. Singh et al., "Tuning the resonance in high-temperature superconducting terahertz metamaterials," Physical Review Letters, vol. 105, no. 24, Article ID 247402, 2010.

[16] R. Singh, A. K. Azad, Q. X. Jia, A. J. Taylor, and H. -T. Chen, "Thermal tunability in terahertz metamaterials fabricated on strontium titanate single-crystal substrates," Optics Letters, vol. 36, no. 7, pp. 1230-1232, 2011.

[17] R. Singh, J. Xiong, A. K. Azad et al., "Optical tuning and ultrafast dynamics of high-temperature superconducting terahertz metamaterials," Nanophotonics. In press, http://arxiv.org/abs/ 1111.3917.

[18] S. Y. Chiam, R. Singh, W. Zhang, and A. A. Bettiol, "Controlling metamaterial resonances via dielectric and aspect ratio effects," Applied Physics Letters, vol. 97, no. 19, Article ID 191906, 2010.

[19] N.-H. Shen, M. Massaouti, M. Gokkavas et al., "Optically implemented broadband blueshift switch in the terahertz regime," Physical Review Letters, vol. 106, no. 3, Article ID 037403, 2011.

[20] D. Roy Chowdhury, R. Singh, J. F. O'Hara, H.-T. Chen, A. J. Taylor, and A. K. Azad, "Dynamically reconfigurable terahertz metamaterial through photo-doped semiconductor," Applied Physics Letters, vol. 99, no. 23, Article ID 231101, 2011.

[21] J. Gu, R. Singh, A. K. Azad et al., "An active hybrid plasmonic metamaterial," Optical Materials Express, vol. 2, Article ID 1617, 2011, http://www.opticsinfobase.org/ome/abstract .cfm? uri=ome-2-1-31.

[22] H.-T. Chen, J. F. O’Hara, A. K. Azad, and A. J. Taylor, "Manipulation of terahertz radiation using metamaterials," Laser \& Photonics Reviews, vol. 5, no. 4, pp. 513-533, 2011.

[23] R. Singh, A. K. Azad, J. F. O’Hara, A. J. Taylor, and W. Zhang, "Effect of metal permittivity on resonant properties of terahertz metamaterials," Optics Letters, vol. 33, no. 13, pp. 1506-1508, 2008.

[24] D. R. Chowdhury, R. Singh, M. Reiten et al., "A broadband planar terahertz metamaterial with nested structure," Optics Express, vol. 19, no. 17, pp. 15817-15823, 2011.

[25] J. F. O’Hara, R. Singh, I. Brener et al., "Thin-film sensing with planar terahertz metamaterials: sensitivity and limitations," Optics Express, vol. 16, no. 3, pp. 1786-1795, 2008.

[26] I. A.I. Al-Naib, C. Jansen, and M. Koch, "Thin-film sensing with planar asymmetric metamaterial resonators," Applied Physics Letters, vol. 93, no. 8, Article ID 083507, 2008.

[27] R. Singh, E. Smirnova, A. J. Taylor, J. F. O’Hara, and W. Zhang, "Optically thin terahertz metamaterials," Optics Express, vol. 16, no. 9, pp. 6537-6543, 2008.
[28] W. Withayachumnankul and D. Abbott, "Metamaterials in the Terahertz Regime,” IEEE Photonics Journal, vol. 1, p. 99, 2009, http://ieeexplore.ieee.org/xpls/abs_all.jsp?arnumber=5130235.

[29] D. R. Chowdhury, R. Singh, M. Reiten, J. Zhou, A. J. Taylor, and J. F. O'Hara, “Tailored resonator coupling for modifying the terahertz metamaterial response," Optics Express, vol. 19, no. 11, pp. 10679-10685, 2011.

[30] F. Hesmer, E. Tatartschuk, O. Zhuromskyy et al., "Coupling mechanisms for split ring resonators: theory and experiment," Physica Status Solidi B, vol. 244, no. 4, pp. 1170-1175, 2007.

[31] R. S. Penciu, K. Aydin, M. Kafesaki et al., "Multi-gap individual and coupled split-ring resonator structures," Optics Express, vol. 16, no. 22, pp. 18131-18144, 2008.

[32] I. Sersic, M. Frimmer, E. Verhagen, and A. F. Koenderink, "Electric and magnetic dipole coupling in near-infrared splitring metamaterial arrays," Physical Review Letters, vol. 103, no. 21, Article ID 213902, 2009.

[33] R. Singh, I. A.I. Al-Naib, Y. Yang et al., "Observing metamaterial induced transparency in individual Fano resonators with broken symmetry," Applied Physics Letters, vol. 99, no. 20, Article ID 201107, 2011.

[34] N. Liu, S. Kaiser, and H. Giessen, "Magnetoinductive and electroinductive coupling in plasmonic metamaterial molecules," Advanced Materials, vol. 20, no. 23, pp. 4521-4525, 2008.

[35] R. Singh, C. Rockstuhl, F. Lederer, and W. Zhang, "Coupling between a dark and a bright eigenmode in a terahertz metamaterial," Physical Review B, vol. 79, no. 8, Article ID 085111, 2009.

[36] N. Feth, M. König, M. Husnik et al., "Electromagnetic interaction of split-ring resonators: the role of separation and relative orientation," Optics Express, vol. 18, no. 7, pp. 6545-6554, 2010.

[37] R. Singh, C. Rockstuhl, F. Lederer, and W. Zhang, "The impact of nearest neighbor interaction on the resonances in terahertz metamaterials," Applied Physics Letters, vol. 94, no. 2, Article ID 021116, 2009.

[38] A. Bitzer, J. Wallauer, H. Helm, H. Merbold, T. Feurer, and M. Walther, "Lattice modes mediate radiative coupling in metamaterial arrays," Optics Express, vol. 17, no. 24, pp. 2210822113, 2009.

[39] R. Singh, C. Rockstuhl, and W. Zhang, "Strong influence of packing density in terahertz metamaterials," Applied Physics Letters, vol. 97, no. 24, Article ID 241108, 2010.

[40] J. Wallauer, A. Bitzer, S. Waselikowski, and M. Walther, "Nearfield signature of electromagnetic coupling in metamaterial arrays: a terahertz microscopy study," Optics Express, vol. 19, no. 18, pp. 17283-17292, 2011.

[41] D. A. Powell, M. Lapine, M. V. Gorkunov, I. V. Shadrivov, and Y. S. Kivshar, "Metamaterial tuning by manipulation of nearfield interaction," Physical Review B, vol. 82, no. 15, Article ID 155128, 2010.

[42] R. Singh, I. A.I. Al-Naib, M. Koch, and W. Zhang, "Sharp Fano resonances in THz metamaterials," Optics Express, vol. 19, no. 7, pp. 6312-6319, 2011.

[43] D. Grischkowsky, S. Keiding, M. van Exter, and C. Fattinger, "Far-infrared time-domain spectroscopy with terahertz beams of dielectrics and semiconductors," Journal of the Optical Society of America B, vol. 7, p. 2006, 1990, http://www .opticsinfobase.org/josab/abstract.cfm?URI=josab-7-10-2006.

[44] Computer Simulation Technology (CST), Darmstadt, Germany.

[45] V. G. Kravets, F. Schedin, and A. N. Grigorenko, "Extremely narrow plasmon resonances based on diffraction coupling 
of localized plasmons in arrays of metallic nanoparticles," Physical Review Letters, vol. 101, no. 8, Article ID 087403, 2008.

[46] C. Rockstuhl, T. Zentgraf, C. Etrich, J. Kuhl, F. Lederer, and H. Giessen, "On the reinterpretation of resonances in split-ringresonators at normal incidence," Optics Express, vol. 14, no. 19, pp. 8827-8836, 2006.

[47] L. Li, "New formulation of the Fourier modal method for crossed surface-relief gratings," Journal of the Optical Society of America A, vol. 14, no. 10, pp. 2758-2767, 1997.

[48] N. Papasimakis, V. A. Fedotov, Y. H. Fu, D. P. Tsai, and N. I. Zheludev, "Coherent and incoherent metamaterials and order-disorder transitions," Physical Review B, vol. 80, no. 4, Article ID 041102, 2009.

[49] C. Helgert, C. Rockstuhl, C. Etrich et al., "Effective properties of amorphous metamaterials," Physical Review B, vol. 79, Article ID 233107, 4 pages, 2009.

[50] R. Singh, X. Lu, J. Gu, Z. Tian, and W. Zhang, "Random terahertz metamaterials," Journal of Optics A, vol. 12, no. 1, Article ID 015101, 2010.

[51] G. Acuna, S. F. Heucke, F. Kuchler, H. T. Chen, A. J. Taylor, and R. Kersting, "Surface plasmons in terahertz metamaterials," Optics Express, vol. 16, no. 23, pp. 18745-18751, 2008.

[52] A. K. Azad, J. Dai, and W. Zhang, "Transmission properties of terahertz pulses through subwavelength double split-ring resonators," Optics Letters, vol. 31, no. 5, pp. 634-636, 2006. 

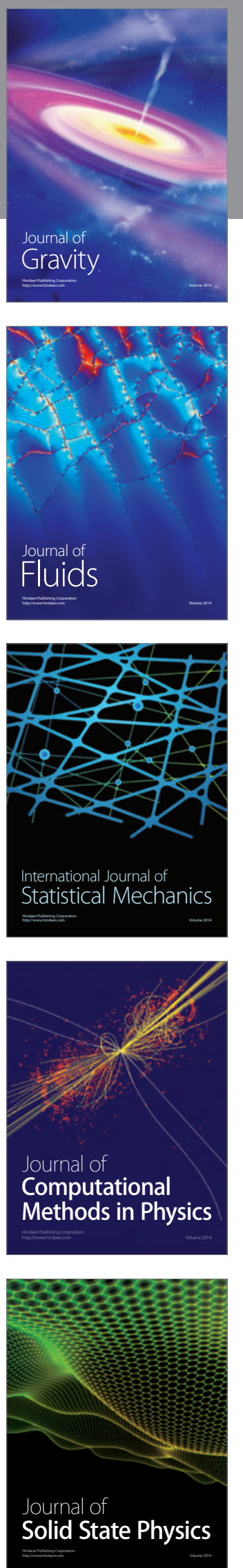

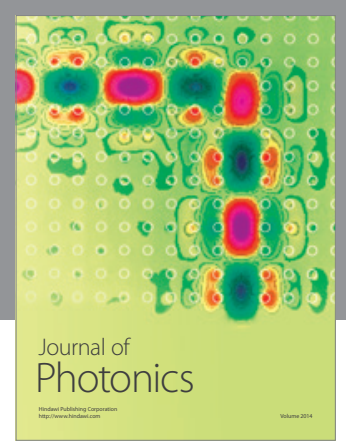

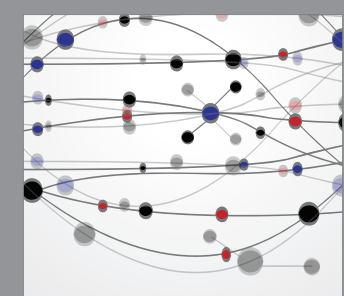

The Scientific World Journal
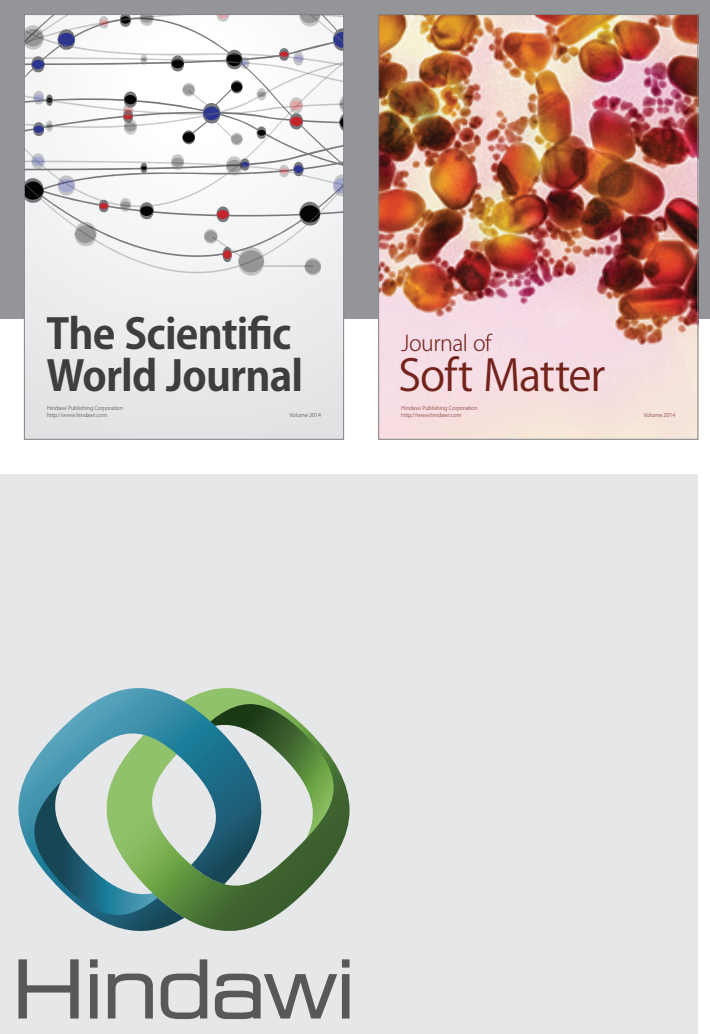

Submit your manuscripts at

http://www.hindawi.com
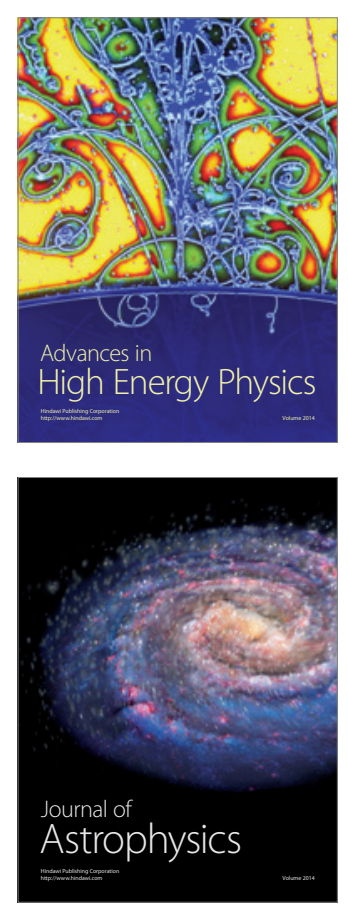
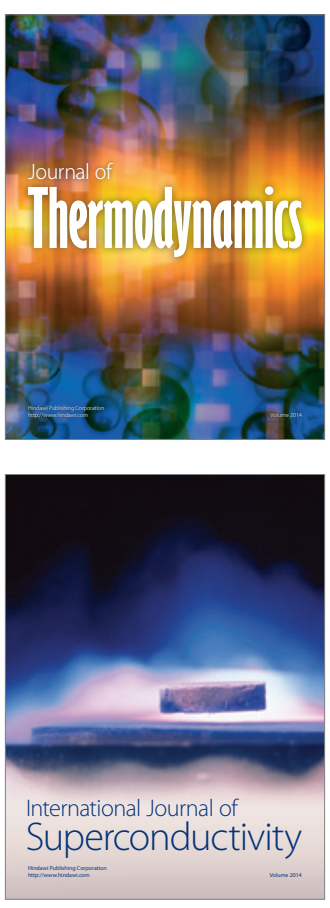
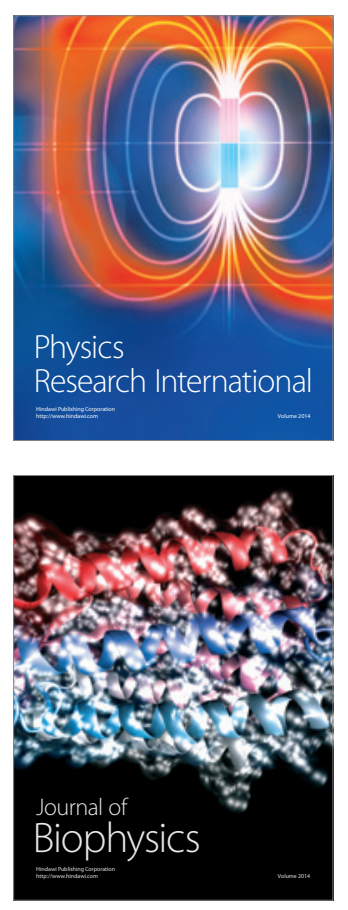
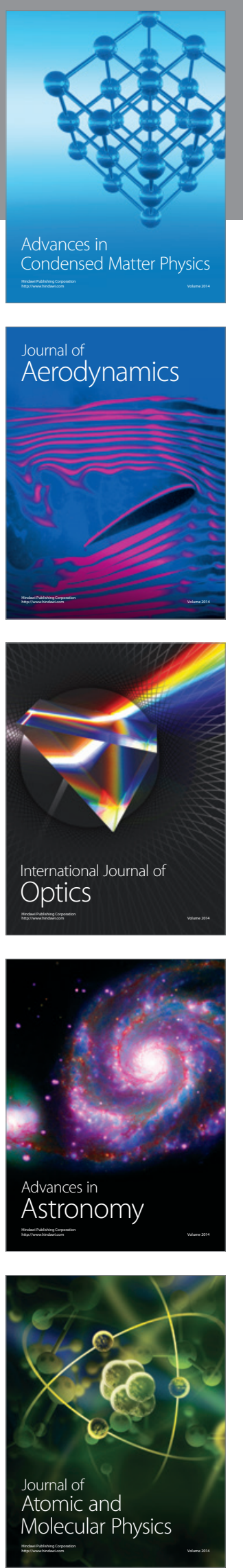